ASC Report No. 08/2007

Improved Entropy Decay Estimates for the Heat Equation

Anton Arnold, Jose Antonio Carrillo, Corinna Klapproth 


\section{Most recent ASC Reports}

07/2007 Christoph Erath, Samuel Ferraz-Leite, Stefan Funken, Dirk Praetorius

Energy Norm Based A Posteriori Error Estimation for Boundary Element Methods in Two Dimensions

06/2007 Anton Arnold, Irene M. Gamba, Maria Pia Gualdani, Christof Sparber The Wigner-Fokker-Planck Equation: Stationary States and Large Time Behavior

05/2007 Anton Arnold, Maike Schulte

Transparent Boundary Conditions for Quantum-Waveguide Simulations

04/2007 Anton Arnold, Jean-Philippe Bartier, Jean Dolbeault

Interpolation between Logarithmic Sobolev and Poincaré Inequalities

03/2007 Tareq Amro, Chunxiong Zheng

A PML Absorbing Boundary Condition for the Nonlinear Euler Equations in Unbounded Domains

02/2007 Christoph Erath, Dirk Praetorius

A Posteriori Error Estimate and Adaptive Mesh-Refinement for the Cell-Centered Finite Volume Method for Elliptic Boundary Value Problems

01/2007 Samuel Ferraz-Leite, Dirk Praetorius

Simple A Posteriori Error Estimators for the h-Version of the Boundary Element Method

Institute for Analysis and Scientific Computing

Vienna University of Technology

Wiedner Hauptstraße 8-10

1040 Wien, Austria

E-Mail: admin@asc.tuwien.ac.at

WWW: http://www.asc.tuwien.ac.at

FAX: $\quad+43-1-58801-10196$

ISBN 978-3-902627-00-1

(C) Alle Rechte vorbehalten. Nachdruck nur mit Genehmigung des Autors.

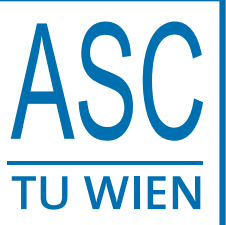




\title{
Improved entropy decay estimates for the heat equation
}

\author{
A. Arnold; J. A. Carrillo, C. Klapproth ${ }^{\ddagger}$
}

April 14, 2007

\begin{abstract}
Improved entropy decay estimates for the heat equation are obtained by selecting well parameterized Gaussians. Either by mass centering or by fixing the second moments or the covariance matrix of the solution, relative entropy towards these Gaussians is shown to decay with better constants than classical estimates.
\end{abstract}

\section{Introduction}

Describing the asymptotic behavior of diffusion and homogeneous kinetic models has recently received a lot of attention in the partial differential equations community [ACDD04]. Several approaches have been established to determine decay estimates towards a distinguished profile for large times. In purely diffusive models, these asymptotic profiles are typically given by self-similar solutions usually coming from stationary solutions of equations in self-similar variables. The use of logarithmic entropies to study large time asymptotics is classical in kinetic theory [Ce82] and it was brought up for diffusion equations in the seminal papers of G. Toscani [To96, To97].

Variations of the entropy-entropy dissipation method connected to the Bakry-Emery strategy [BE84, BE85] have been used to describe these rates for linear and nonlinear diffusion equations [AMTU01, CT00, CJMTU01]. Deep connections to optimal transport issues were discovered by F. Otto [Ot01]. He obtained these decay estimates using a suitable interpretation of the diffusion equations as gradient flows/steepest descent of entropy or free-energy functionals with respect to a formal Riemannian structure inducing an optimal transport distance. These decay estimates in some cases were already known by classical techniques involving compactness, scalings and maximum principle arguments [Ba97, Va03, Va06]. In all these works, the decay estimates have been obtained in different senses, mainly: entropy decay [AMTU01, CT00, Ot01, CJMTU01], optimal transport distance decay [Ot01], and $L^{1}$-decay [Va03], and in [CT00, Ot01, CJMTU01] as a consequence of Csiszár-Kullback type inequalities [Cs63, Ku59, UAMT00].

Once the first asymptotic term has been pinpointed, the next step is to improve the decay rate either by taking into account other invariances of the equation or by identifying

\footnotetext{
${ }^{*}$ Institut für Analysis und Scientific Computing, E101, Technische Universität Wien, Wiedner Hauptstr. 8, A-1040 Wien, Austria. E-mail: anton.arnold@tuwien.ac.at

${ }^{\dagger}$ ICREA (Institució Catalana de Recerca i Estudis Avançats) and Departament de Matemàtiques, Universitat Autònoma de Barcelona, E-08193 Bellaterra, Spain. E-mail: carrillo@mat.uab.es

${ }^{\ddagger}$ ZIB, Takustraße 7, D-14195 Berlin, Germany. E-mail: klapproth@zib.de
} 
the next term in the large-time asymptotic expansion. In the case of the heat equation, expansion at all orders of the solutions for large times in $L^{1}$ were obtained in [DZ92]. More precisely, as long as more and more moments of the initial data are bounded, a better approximation in terms of derivatives of the fundamental solution of the heat equation and the moments of the initial data can be given for large times. A similar result without identifying the asymptotic expansion at all orders was obtained in [GJT02] by using Fourier-based distances.

Obtaining the next terms in the asymptotic expansion and identifying the corresponding improved decay estimates and rates are interesting and important open question in the nonlinear diffusion case. This question has been addressed recently for the fast diffusion equation at the linearized level [DM04] and, finally, proved for the nonlinear fast-diffusion equation in [KM05, KM07, MS06]. These results take advantage of the complete knowledge of the spectrum of the linearized operator: first they show that solutions will lie for large times in a neighborhood of an asymptotic profile and then, they try from the linearized improved decay rates to infer the result over the nonlinear one. In particular, they show that mass-centering speeds up the convergence rate for different particular cases of the diffusion exponent. In the case of the porous medium equation, a formal expansion to all orders of the solutions in the one-dimensional case was done in [An88]. Finally, the improvement of decay rates and decay estimates for the porous medium equation by either mass-centering of by fixing equal variance was discussed in [WB98]. Essentially, these results give decay improvements in $L^{1}$-spaces. An improvement on the optimal transport distance decay by mass centering has been reported in the one dimensional case [CDT07].

Here, we will show how the entropy decay estimates for the heat equation can be improved by mass-centering and by fixing the covariance matrix of the approximations. In section 2, an improved decay estimate for the heat equation by fixing center of mass and variance is obtained, whereas section 3 is devoted to generalize this idea in the case of fixing the whole covariance matrix of the approximated Gaussian. Let us finally mention that these improvements will be at the level of the constants in the decay estimates but not at the level of the decay rates for large times. Although the improvement of the decay rate is expected and true at the $L^{1}$ level [DZ92], the present approach does not yield it for the relative entropy.

\section{The heat equation and isotropic Gaussians}

It is well-known that solutions of the Cauchy problem for the heat equation with diffusion constant $k / 2$ :

$$
\begin{cases}\frac{\partial u}{\partial t}=\frac{k}{2} \triangle u, & x \in \mathbb{R}^{n}, t>0 \\ u(x, t=0)=u_{0}(x), & x \in \mathbb{R}^{n}\end{cases}
$$

behave asymptotically like a Gaussian with the same mass as the solution and a variance that is linearly increasing in $t$. This result can be easily recovered from the classical logarithmic Sobolev inequality (LSI) of Gross [Gr75, AMTU01] in $\mathbb{R}^{n}$ with respect to the isotropic Gaussian measure

$$
d M_{\sigma}=(2 \pi \sigma)^{-\frac{n}{2}} e^{-\frac{|x|^{2}}{2 \sigma}} d x
$$

as in [To96] guided by classical arguments from kinetic theory. Let us quickly review a simplified proof of the one given in [To96]. Consider any two probability densities $\rho_{1}, \rho_{2}$ 
on $\mathbb{R}^{n}$, i.e. $\rho_{1}, \rho_{2} \in L_{+}^{1}\left(\mathbb{R}^{n}\right)$ with

$$
\int_{\mathbb{R}^{n}} \rho_{1} d x=\int_{\mathbb{R}^{n}} \rho_{2} d x=1 .
$$

We define the relative logarithmic entropy of $\rho_{1}$ w.r.t. $\rho_{2}$ as

$$
e_{1}\left(\rho_{1} \mid \rho_{2}\right):=\int_{\mathbb{R}^{n}} \frac{\rho_{1}}{\rho_{2}} \ln \frac{\rho_{1}}{\rho_{2}} \rho_{2} d x \geq 0,
$$

and $\int \rho_{1} \ln \rho_{1} d x$ is the logarithmic entropy of the probability density $\rho_{1}$. The LSI w.r.t. the Gaussian measure $M_{\sigma}$ reads [Gr75]

$$
\int_{\mathbb{R}^{n}} g^{2} \ln g^{2} d M_{\sigma} \leq 2 \sigma \int_{\mathbb{R}^{n}}|\nabla g|^{2} d M_{\sigma}
$$

for all $\sigma>0$ and $g \in L^{2}\left(\mathbb{R}^{n}, d M_{\sigma}\right)$ with $\int g^{2} d M_{\sigma}=1$. By setting $g^{2}=\frac{\rho}{M_{\sigma}}$ it is equivalent to

$$
e_{1}\left(\rho \mid M_{\sigma}\right) \leq \frac{\sigma}{2} I\left(\rho \mid M_{\sigma}\right)
$$

for all $\rho \in L_{+}^{1}\left(\mathbb{R}^{n}\right)$ with $\int \rho d x=1$. Here,

$$
I\left(\rho_{1} \mid \rho_{2}\right):=\int_{\mathbb{R}^{n}} \frac{\rho_{2}}{\rho_{1}}\left|\nabla\left(\frac{\rho_{1}}{\rho_{2}}\right)\right|^{2} \rho_{2} d x=\int_{\mathbb{R}^{n}}\left|\nabla \ln \left(\frac{\rho_{1}}{\rho_{2}}\right)\right|^{2} \rho_{1} d x=4 \int_{\mathbb{R}^{n}}\left|\nabla \sqrt{\frac{\rho_{1}}{\rho_{2}}}\right|^{2} \rho_{2} d x \geq 0
$$

denotes the relative Fisher information of $\rho_{1}$ w.r.t. $\rho_{2}$ [Fi25]. There is equality in (2.3) if and only if

$$
g(x)=g_{y}(x):=\exp \left(\frac{x \cdot y}{\sqrt{\sigma}}-|y|^{2}\right)
$$

for an arbitrary $y \in \mathbb{R}^{n}$, as established by Carlen in [Ca91].

Theorem 2.1 (Standard Decay Estimate) [To96] Let the initial value for the heat equation $u_{0} \in C\left(\mathbb{R}^{n}\right) \cap W_{\text {loc }}^{1,2}\left(\mathbb{R}^{n}\right) \cap L_{+}^{1}\left(\mathbb{R}^{n}\right)$ be a probability density on $\mathbb{R}^{n}$ with finite second moment and entropy, i.e. $u_{0}(x) \geq 0, \int u_{0} d x=1, \int|x|^{2} u_{0} d x<\infty$, and $\int u_{0}\left|\ln u_{0}\right| d x<$ $\infty$. Then, the relative logarithmic entropy of the solution $u$ to (2.1) w.r.t. $u_{\infty}(x, t):=$ $M_{E+k t}\left(x-\tilde{x}_{0}\right)$ with an arbitrary $\tilde{x}_{0} \in \mathbb{R}^{n}$ and an arbitrary $E>0$ satisfies the decay estimate

$$
e_{1}\left(u(t) \mid u_{\infty}(t)\right) \leq \frac{E}{E+k t} e_{1}\left(u_{0} \mid u_{\infty}(0)\right), \quad \forall t \geq 0 .
$$

Proof. Since $u$ and $u_{\infty}$ are solutions of the heat equation, they are smooth, positive, and rapidly decaying functions for all $t>0$. Thus, we find for all $t>0$

$$
\begin{aligned}
\frac{d}{d t} e_{1}\left(u(t) \mid u_{\infty}(t)\right) & =\int_{\mathbb{R}^{n}} \frac{\partial u}{\partial t}\left[\ln \left(\frac{u}{u_{\infty}}\right)+1\right] d x-\int_{\mathbb{R}^{n}} \frac{\partial u_{\infty}}{\partial t} \frac{u}{u_{\infty}} d x \\
& =\frac{k}{2} \int_{\mathbb{R}^{n}} \triangle u\left[\ln \left(\frac{u}{u_{\infty}}\right)+1\right] d x-\frac{k}{2} \int_{\mathbb{R}^{n}} \triangle u_{\infty} \frac{u}{u_{\infty}} d x \\
& =-\frac{k}{2} \int_{\mathbb{R}^{n}} \frac{u_{\infty}}{u} \nabla u \cdot \nabla\left(\frac{u}{u_{\infty}}\right) d x+\frac{k}{2} \int_{\mathbb{R}^{n}} \nabla u_{\infty} \cdot \nabla\left(\frac{u}{u_{\infty}}\right) d x \\
& =-\frac{k}{2} I\left(u(t) \mid u_{\infty}(t)\right) .
\end{aligned}
$$


Hence, $e_{1}\left(u(, t) \mid u_{\infty}(t)\right)$ is non-increasing in time. Conservation of mass for the heat equation shows that $u$ is a probability density for $t>0$. And since $u_{\infty}(t)$ is a Gaussian with second moment $n(E+k t)$, we infer from the LSI (2.3) that

$$
e_{1}\left(u(t) \mid u_{\infty}(t)\right) \leq \frac{E+k t}{2} I\left(u(t) \mid u_{\infty}(t)\right) .
$$

Applying this bound to the right-hand side of (2.8) yields with Gronwall's lemma

$$
e_{1}\left(u(t) \mid u_{\infty}(t)\right) \leq \frac{E}{E+k t} e_{1}\left(u_{0} \mid u_{\infty}(0)\right), \quad \forall t \geq 0 .
$$

Since

$$
\begin{aligned}
e_{1}\left(u_{0} \mid u_{\infty}(0)\right)= & \int_{\mathbb{R}^{n}} u_{0}(x) \ln u_{0}(x) d x+\frac{n}{2} \ln (2 \pi(E+k t)) \\
& +\frac{1}{2(E+k t)} \int_{\mathbb{R}^{n}}\left|x-\tilde{x}_{0}\right|^{2} u_{0}(x) d x<\infty
\end{aligned}
$$

we conclude that $u$ converges in logarithmic entropy to $u_{\infty}$ as $t \rightarrow \infty$.

Remark 2.2 (Sharpness) Due to the translational invariance of the heat equation and the equality cases (2.6) of the LSI, the decay estimate (2.7) is sharp in the following sense: choosing $u_{0}(x)=M_{E}\left(x-\tilde{x}_{0}^{\prime}\right)$ for an arbitrary $\tilde{x}_{0}^{\prime} \in \mathbb{R}^{n}$, the solution of the Cauchy problem for the heat equation is $u(x, t)=M_{E+k t}\left(x-\tilde{x}_{0}^{\prime}\right)$ and

$$
e_{1}\left(u(\cdot, t) \mid M_{E+k t}\left(\cdot-\tilde{x}_{0}\right)\right)=\frac{\left|\tilde{x}_{0}-\tilde{x}_{0}^{\prime}\right|^{2}}{2(E+k t)}, \quad \forall t \geq 0,
$$

leading to non-trivial equality in (2.7).

On the way to improving the decay rate in relative entropy for the solution of (2.1) we shall compare $u(t)$ to a better fitted Gaussian $M_{\sigma(t)}$ - rather than to $M_{E+k t}$. Solutions of the heat equation obviously conserve the center of mass:

$$
\int_{\mathbb{R}^{n}} x u(x, t) d x=\int_{\mathbb{R}^{n}} x u_{0}(x) d x:=x_{0}, \quad \forall t>0,
$$

and linearly increase the second moment:

$$
\int_{\mathbb{R}^{n}}\left|x-x_{0}\right|^{2} u(x, t) d x=\int_{\mathbb{R}^{n}}\left|x-x_{0}\right|^{2} u_{0}(x) d x+n k t:=\alpha+n k t, \quad \forall t>0 .
$$

Our first observation is that

$$
e_{1}\left(u(\cdot, t) \mid M_{\frac{\alpha}{n}+k t}\left(\cdot-x_{0}\right)\right)=\min _{\substack{E>0 \\ \tilde{x}_{0} \in \mathbb{R}^{n}}} e_{1}\left(u(\cdot, t) \mid M_{E+k t}\left(\cdot-\tilde{x}_{0}\right)\right),
$$

as it can easily be checked just by working on the explicit expression of the relative entropy $e_{1}\left(u(\cdot, t) \mid M_{E+k t}\left(\cdot-\tilde{x}_{0}\right)\right)$. In other words, the optimal Gaussian (in the sense of minimizing the relative entropy) for a given solution $u$ at a fixed time $t \geq 0$ is given by the Gaussian with the same center of mass and variance as $u$, i.e. $M_{\frac{\alpha}{n}+k t}\left(x-x_{0}\right)$. 
In the following, we want to discuss if the decay estimate (2.7) for the relative entropy of $u$ w.r.t. to the optimal Gaussian $M_{\frac{\alpha}{n}+k t}\left(x-x_{0}\right)$ given by

$$
e_{1}\left(u(\cdot, t) \mid M_{\frac{\alpha}{n}+k t}\left(\cdot-x_{0}\right)\right) \leq \frac{\frac{\alpha}{n}}{\frac{\alpha}{n}+k t} e_{1}\left(u_{0} \mid M_{\frac{\alpha}{n}}\left(\cdot-x_{0}\right)\right), \quad t \geq 0
$$

is sharp as well. Clearly, we have equality for

$$
u_{0}(x)=M_{\frac{\alpha}{n}}\left(x-x_{0}\right),
$$

but then both sides of (2.13) are zero. For other cases, equality in (2.13) for all $t \geq 0$ would imply equality at $t=0$ of both $t$-derivatives, i.e. $-\frac{k}{2} I=-\frac{n}{\alpha} k e_{1}$. But this is the LSI (2.4), which becomes an equality only for the shifted Gaussians (2.6). But then, the equality of moments in the optimized Gaussian (cf. (2.12)) leaves (2.14) as the only case. Hence, there exists no initial function $u_{0}$ satisfying the conditions of (2.12) such that there is non-zero equality in (2.13) and this decay estimate is not sharp anymore.

Now, we come back to Theorem 2.1 and the estimate

$$
e_{1}\left(u(\cdot, t) \mid M_{E+k t}\left(\cdot-\tilde{x}_{0}\right)\right) \leq \frac{E}{E+k t} e_{1}\left(u_{0} \mid M_{E}\left(\cdot-\tilde{x}_{0}\right)\right)
$$

for an arbitrary $\tilde{x}_{0} \in \mathbb{R}^{n}$ and an arbitrary $E>0$. We observe that for small values $E>0$ the ratio $E /(E+k t)$ decays faster to zero as $t$ goes to infinity than for large $E$ 's. This leads us to the conjecture that it is possible to find a sharper estimate for the logarithmic entropy of the solution $u$ w.r.t. the Gaussian $M_{\frac{\alpha}{n}+k t}\left(\cdot-x_{0}\right)$ than $(2.13)$ by determining a function $E(t)$ with $0<E(t)<\frac{\alpha}{n}$ for $t>0$, which should be used instead of a constant $E$ on the right-hand side of (2.15).

The idea for deriving such an optimized decay estimate is to minimize the right-hand side of inequality (2.15) w.r.t. $E>0$ and $\tilde{x}_{0} \in \mathbb{R}^{n}$. As before, we find for all fixed times $t \geq 0$ that

$$
\begin{aligned}
e_{1}\left(u(\cdot, t) \mid M_{\frac{\alpha}{n}+k t}\left(\cdot-x_{0}\right)\right) & =\min _{\substack{E>0 \\
\tilde{x}_{0} \in \mathbb{R}^{n}}} e_{1}\left(u(\cdot, t) \mid M_{E+k t}\left(\cdot-\tilde{x}_{0}\right)\right) \\
& \leq \inf _{\substack{E>0 \\
\tilde{x}_{0} \in \mathbb{R}^{n}}} \frac{E}{E+k t} e_{1}\left(u_{0} \mid M_{E}\left(\cdot-\tilde{x}_{0}\right)\right) \\
& =\inf _{E>0} \frac{E}{E+k t} e_{1}\left(u_{0} \mid M_{E}\left(\cdot-x_{0}\right)\right) .
\end{aligned}
$$

In the case $u_{0}(x)=M_{\frac{\alpha}{n}}\left(x-x_{0}\right)$, we have $e_{1}\left(u(\cdot, t) \mid M_{\frac{\alpha}{n}+k t}\left(\cdot-x_{0}\right)\right)=0$ for all times $t \geq 0$ and it holds equality in the estimate (2.7). Hence, we obtain the minimum of the righthand side of (2.16) and therefore the best estimate for the choice $E=\frac{\alpha}{n}$. In general we have to determine the time-dependent second moment $E(t)>0$ by minimizing for each fixed $t>0$ the function $f: \mathbb{R}^{+} \times \mathbb{R}_{0}^{+} \longrightarrow \mathbb{R}_{0}^{+}$defined as

$$
\begin{aligned}
f(E, t) & :=\frac{E}{E+k t} e_{1}\left(u_{0} \mid M_{E}\left(\cdot-x_{0}\right)\right) \\
& =\frac{E}{E+k t}\left(\int_{\mathbb{R}^{n}} u_{0}(x) \ln u_{0}(x) d x+\frac{n}{2} \ln (2 \pi E)+\frac{\alpha}{2 E}\right)
\end{aligned}
$$

which leads us to the following result: 
Lemma 2.3 (Computation of $E_{\text {min }}(t)$ ) Let $u_{0}(x) \neq M_{\frac{\alpha}{n}}\left(x-x_{0}\right)$ on a set of positive measure. Then the function $f(E, t)$ defined by (2.17) has w.r.t. $E>0$ for all fixed $t \geq 0$ a unique minimum $E_{\min }(t)$ with the following properties:

a) $E_{\min }(t)$ satisfies

$$
\left(\int_{\mathbb{R}^{n}} u_{0}(x) \ln u_{0}(x) d x+\frac{n}{2} \ln \left(2 \pi E_{\min }(t)\right)+\frac{n}{2}\right) k t=\frac{\alpha}{2}-\frac{n}{2} E_{\min }(t), \quad \forall t \geq 0
$$

b) $E_{\min }(0)=\frac{\alpha}{n}$

c) $E_{\text {min }}^{\infty}:=\lim _{t \rightarrow \infty} E_{\text {min }}(t)=\frac{\alpha}{n} \exp \left(-\frac{2}{n} e_{1}\left(u_{0} \mid M_{\frac{\alpha}{n}}\left(\cdot-x_{0}\right)\right)\right)$

$$
=\frac{1}{2 \pi} \exp \left(-\frac{2}{n} \int_{\mathbb{R}^{n}} u_{0}(x) \ln u_{0}(x) d x-1\right)
$$

d) $E_{\min }(t)$ is strictly monotonic decreasing w.r.t. $t \geq 0$. In particular,

$$
0<E_{\min }(t)<\frac{\alpha}{n}, \quad \forall t>0
$$

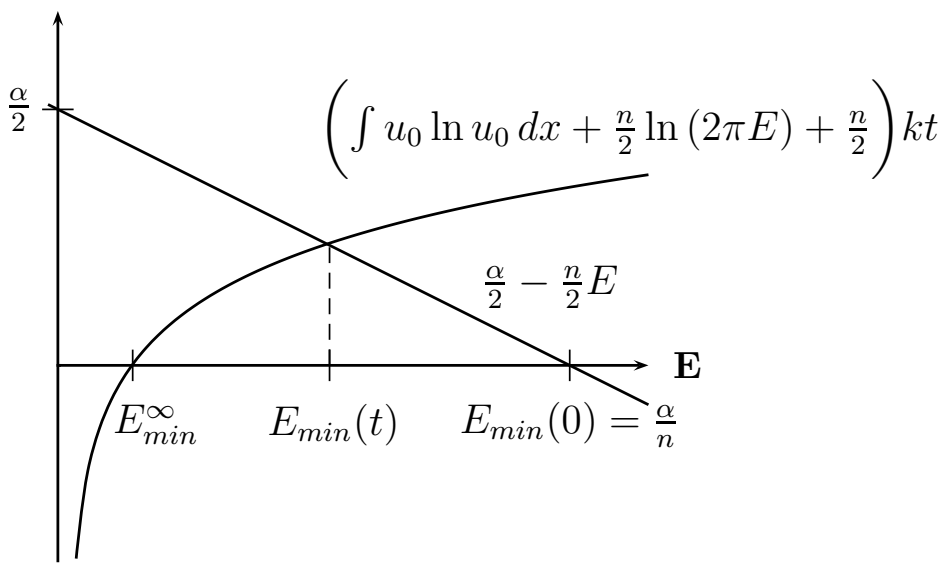

Figure 1: $E_{\min }(t)$ is the intersection point of the functions on the left-hand and right-hand side of $(2.18)$ for all $t \geq 0 . \lim _{t \rightarrow \infty} E_{\text {min }}(t)=E_{\text {min }}^{\infty}$ and $E_{\min }(0)=\frac{\alpha}{n}$ are the unique roots of the left- and right-hand side of (2.18).

Proof. $f(E, t)$ is for all times $t \geq 0$ differentiable w.r.t. $E$ with

$$
\begin{aligned}
\frac{\partial}{\partial E} f(E, t) & \left.=\frac{k t}{(E+k t)^{2}} e_{1}\left(u_{0}\right) \mid M_{E}\left(\cdot-x_{0}\right)\right)+\frac{E}{E+k t}\left(\frac{n}{2 E}-\frac{\alpha}{2 E^{2}}\right) \\
& =\frac{k t}{(E+k t)^{2}}\left(\int_{\mathbb{R}^{n}} u_{0} \ln u_{0} d x+\frac{n}{2} \ln (2 \pi E)\right)+\frac{n}{2(E+k t)}-\frac{\alpha}{2(E+k t)^{2}} \\
& =\frac{\tilde{f}(E, t)}{(E+k t)^{2}}
\end{aligned}
$$

where

$$
\tilde{f}(E, t)=\left(\int_{\mathbb{R}^{n}} u_{0} \ln u_{0} d x+\frac{n}{2} \ln (2 \pi E)\right) k t+\frac{n}{2}(E+k t)-\frac{\alpha}{2}
$$


is monotonously increasing w.r.t. $E>0$. We observe that $\tilde{f}(E, t)$ converges to $-\infty$ as $E \longrightarrow 0+$ and evaluating the function at the point $E=\frac{\alpha}{n}$ leads to

$$
\tilde{f}\left(E=\frac{\alpha}{n}, t\right)=e_{1}\left(u_{0} \mid M_{\frac{\alpha}{n}}\left(\cdot-x_{0}\right)\right) \cdot k t>0, \quad \forall t>0 .
$$

We conclude by the continuity of $\tilde{f}(E, t)$ w.r.t. $E>0$ and the intermediate value theorem that $\tilde{f}(E, t)$ is zero at one point $E_{\min }(t) \in\left(0, \frac{\alpha}{n}\right)$ and thus, $\tilde{f}(E, t)$ and $\partial_{E} f(E, t)$ have a unique zero for $t>0$. The fact that $\tilde{f}(E, t)$ and $\partial_{E} f(E, t)$ have the same sign yields the uniqueness of a minimum $E_{\min }(t)$ in $\left(0, \frac{\alpha}{n}\right)$ of $f(E, t)$ for $t>0$.

a) By setting (2.19) to zero and rewriting the equation we find for all $t \geq 0$ a condition for $E_{\min }(t)$ such that of $\partial_{E} f(E, t)$ becomes zero:

$$
\left(\int_{\mathbb{R}^{n}} u_{0} \ln u_{0} d x+\frac{n}{2} \ln \left(2 \pi E_{\min }(t)\right)+\frac{n}{2}\right) k t=\frac{\alpha}{2}-\frac{n}{2} E_{\min }(t) .
$$

b) Evaluating (2.20) at $t=0$ yields $E_{\min }(0)=\frac{\alpha}{n}$.

c) Since $E_{\min }(t)$ is bounded we find by $(2.20)$ that $E_{\min }^{\infty}:=\lim _{t \rightarrow \infty} E_{\min }(t)$ solves the equation

$$
\int_{\mathbb{R}^{n}} u_{0} \ln u_{0} d x+\frac{n}{2} \ln \left(2 \pi E_{m i n}^{\infty}\right)+\frac{n}{2}=0,
$$

which is equivalent to

$$
e_{1}\left(u_{0} \mid M_{\frac{\alpha}{n}}\left(\cdot-x_{0}\right)\right)-\frac{n}{2} \ln \left(2 \pi \frac{\alpha}{n}\right)+\frac{n}{2} \ln \left(2 \pi E_{\text {min }}^{\infty}\right)=0 .
$$

d) Differentiating the expression (2.20) w.r.t. $t \geq 0$ gives

$$
E_{\text {min }}^{\prime}(t)=-\frac{E_{\min }(t)}{E_{\min }(t)+k t} \frac{2}{n t}\left(\frac{\alpha}{2}-\frac{n}{2} E_{\min }(t)\right), \quad \forall t>0 .
$$

Since $E_{\min }(t) \in\left(0, \frac{\alpha}{n}\right)$ for $t>0$, we have $E_{\min }^{\prime}(t)<0$ for $t>0$ and

$$
E_{\min }^{\prime}(0)=-\frac{2 k}{n} e_{1}\left(u_{0} \mid M_{\frac{\alpha}{n}}\left(\cdot-x_{0}\right)\right)<0 .
$$

Thus, $E_{\min }(t)$ is strictly monotonic decreasing w.r.t. $t \geq 0$.

This concludes the proof.

In the case $u_{0}(x)=M_{\frac{\alpha}{n}}\left(x-x_{0}\right)$ a.e., we define $E_{\text {min }}(t):=\frac{\alpha}{n}$. Using Lemma 2.3 together with (2.16) we can now improve the decay estimate for the relative entropy in Theorem 2.1: 
Theorem 2.4 (Improved Decay Estimate) Let the initial value $u_{0} \in C\left(\mathbb{R}^{n}\right) \cap W_{\text {loc }}^{1,2}\left(\mathbb{R}^{n}\right)$ $\cap L_{+}^{1}\left(\mathbb{R}^{n}\right)$ be a probability density on $\mathbb{R}^{n}$ with finite second moment and finite absolute entropy, i.e. $u_{0} \geq 0, \int u_{0} d x=1, \int|x|^{2} u_{0} d x<\infty$, and $\int u_{0}\left|\ln u_{0}\right| d x<\infty$. Then the solution $u$ of the initial value problem (IVP) (2.1) satisfies

$$
\begin{aligned}
e_{1}\left(u(\cdot, t) \mid M_{\frac{\alpha}{n}+k t}\left(\cdot-x_{0}\right)\right) & \leq \frac{E_{\min }(t)}{E_{\min }(t)+k t} e_{1}\left(u_{0} \mid M_{E_{\min }(t)}\left(\cdot-x_{0}\right)\right) \\
& =\frac{1}{k t}\left(\frac{\alpha}{2}-\frac{n}{2} E_{\min }(t)\right), \quad \forall t>0 .
\end{aligned}
$$

Proof. By (2.16) we obtain the optimized estimate

$$
e_{1}\left(u(\cdot, t) \mid M_{\frac{\alpha}{n}+k t}\left(\cdot-x_{0}\right)\right) \leq f\left(E_{\min }(t), t\right) .
$$

Inserting the conditional equation $(2.20)$ for $E_{\min }(t)$ in the definition of $f(E, t)$ gives

$$
\begin{aligned}
f\left(E_{\min }(t), t\right) & =\frac{E_{\min }(t)}{E_{\min }(t)+k t} e_{1}\left(u_{0} \mid M_{E_{\text {min }}(t)}\left(\cdot-x_{0}\right)\right) \\
& =\frac{E_{\min }(t)}{E_{\min }(t)+k t}\left[\frac{1}{k t}\left(\frac{\alpha}{2}-\frac{n}{2} E_{\min }(t)\right)-\frac{n}{2}+\frac{\alpha}{2 E_{\min }(t)}\right] \\
& =\frac{E_{\min }(t)}{E_{\min }(t)+k t}\left(\frac{1}{k t}+\frac{1}{E_{\min }(t)}\right)\left(\frac{\alpha}{2}-\frac{n}{2} E_{\min }(t)\right) \\
& =\frac{1}{k t}\left(\frac{\alpha}{2}-\frac{n}{2} E_{\min }(t)\right)
\end{aligned}
$$

concluding the proof.

We end this section by analyzing the sharpness of the optimized decay estimate (2.23) from Theorem 2.4. For some fixed initial value $u_{0}$ let us assume that there holds equality in (2.23) on some (possibly small) time interval $[0, T]$, i.e.

$e_{1}\left(u(\cdot, t) \mid M_{\frac{\alpha}{n}+k t}\left(\cdot-x_{0}\right)\right)=\frac{E_{\min }(t)}{E_{\min }(t)+k t}\left(\int_{\mathbb{R}^{n}} u_{0} \ln u_{0} d x+\frac{n}{2} \ln \left(2 \pi E_{\min }(t)\right)+\frac{\alpha}{2 E_{\min }(t)}\right)$.

Differentiating this equality w.r.t. the time $t \geq 0$ yields

$$
\begin{aligned}
& \frac{d}{d t} e_{1}\left(u(\cdot, t) \mid M_{\frac{\alpha}{n}+k t}\left(\cdot-x_{0}\right)\right) \\
& \quad=\frac{E_{\min }^{\prime}(t) k t-E_{\min }(t) k}{\left(E_{\min }(t)+k t\right)^{2}} e_{1}\left(u_{0} \mid M_{E_{\min }(t)}\left(\cdot-x_{0}\right)\right)+\frac{E_{\min }^{\prime}(t)}{E_{\min }(t)+k t}\left(\frac{n}{2}-\frac{\alpha}{2 E_{\min }(t)}\right) .
\end{aligned}
$$

From (2.8) we know that for all times $t \geq 0$

$$
\frac{d}{d t} e_{1}\left(u(\cdot, t) \mid M_{\frac{\alpha}{n}+k t}\left(\cdot-x_{0}\right)\right)=-\frac{k}{2} I\left(u(\cdot, t) \mid M_{\frac{\alpha}{n}+k t}\left(\cdot-x_{0}\right)\right) .
$$

Evaluating (2.24) at time $t=0$ gives with $E_{\min }(0)=\frac{\alpha}{n}$ :

$$
\frac{\alpha}{2 n} I\left(u_{0} \mid M_{\frac{\alpha}{n}}\left(\cdot-x_{0}\right)\right)=e_{1}\left(u_{0} \mid M_{\frac{\alpha}{n}}\left(\cdot-x_{0}\right)\right) .
$$


This LSI becomes an equality only in the case (2.6), where

$$
g^{2}(x):=\frac{u_{0}(x)}{M_{\frac{\alpha}{n}}\left(x-x_{0}\right)} .
$$

Finally, we find with $t=0$ and $E=\frac{\alpha}{n}$ for the normalized $u_{0}$ the condition

$$
u_{0}(x)=M_{\frac{\alpha}{n}}\left(x-\left(x_{0}+2 \sqrt{\frac{\alpha}{n}} y\right)\right)
$$

where $y \in \mathbb{R}^{n}$ is arbitrary. Since the first moment of $u_{0}$ is assumed to be equal to $x_{0}$, we conclude that

$$
u_{0}(x)=M_{\frac{\alpha}{n}}\left(x-x_{0}\right) .
$$

Hence, we have equality in (2.23) only in the case that both sides of the inequality are equal to zero. So, the optimized decay estimate is not sharp in the sense above.

Next we compare our improved decay estimate (2.23) to the classical estimate (2.13) by Toscani [To96]. Let us first discuss their large-time behaviors. Actually, (2.18) is equivalent to

$$
\begin{aligned}
E_{\text {min }}(t) & =\frac{1}{2 \pi} \exp \left[-\frac{2}{n} \int_{\mathbb{R}^{n}} u_{0} \ln u_{0} d x-1+\frac{2}{n k t}\left(\frac{\alpha}{2}-\frac{n}{2} E_{\text {min }}(t)\right)\right] \\
& =E_{\text {min }}^{\infty} \exp \left[\frac{2}{n k t}\left(\frac{\alpha}{2}-\frac{n}{2} E_{\text {min }}(t)\right)\right], \quad \forall t>0,
\end{aligned}
$$

that can be expanded for $t \gg 1$ :

$$
E_{\min }(t)=E_{\min }^{\infty}\left[1+\frac{2}{n k t}\left(\frac{\alpha}{2}-\frac{n}{2} E_{\min }(t)\right)+\mathcal{O}\left(t^{-2}\right)\right] .
$$

Thus, the quantity $E_{\min }(t)-E_{m i n}^{\infty}$ is for large times $t$ proportional to $\left(E_{m i n}^{\infty}+k t\right)^{-1}$ and we obtain for the improved decay estimate the approximation

$$
\begin{aligned}
e_{1}\left(u(\cdot, t) \mid M_{\frac{\alpha}{n}+k t}\left(\cdot-x_{0}\right)\right) & \leq \frac{1}{k t}\left[\frac{\alpha}{2}-\frac{n}{2} E_{\text {min }}^{\infty}-\left(\frac{\alpha}{2}-\frac{n}{2} E_{\text {min }}^{\infty}\right) \frac{E_{\text {min }}^{\infty}}{E_{\text {min }}^{\infty}+k t}+\mathcal{O}\left(t^{-2}\right)\right] \\
& =\frac{\frac{\alpha}{2}-\frac{n}{2} E_{\text {min }}^{\infty}}{E_{\text {min }}^{\infty}+k t}+\mathcal{O}\left(t^{-3}\right), \quad \forall t \gg 1 .
\end{aligned}
$$

The large time behavior of our new decay estimate is similar to the original decay estimate: The right-hand side of $(2.23)$ is proportional to $\left(E_{\min }^{\infty}+k t\right)^{-1}$ for large times $t$ while the estimate $(2.13)$ is proportional to $\left(\frac{\alpha}{n}+k t\right)^{-1}$. 

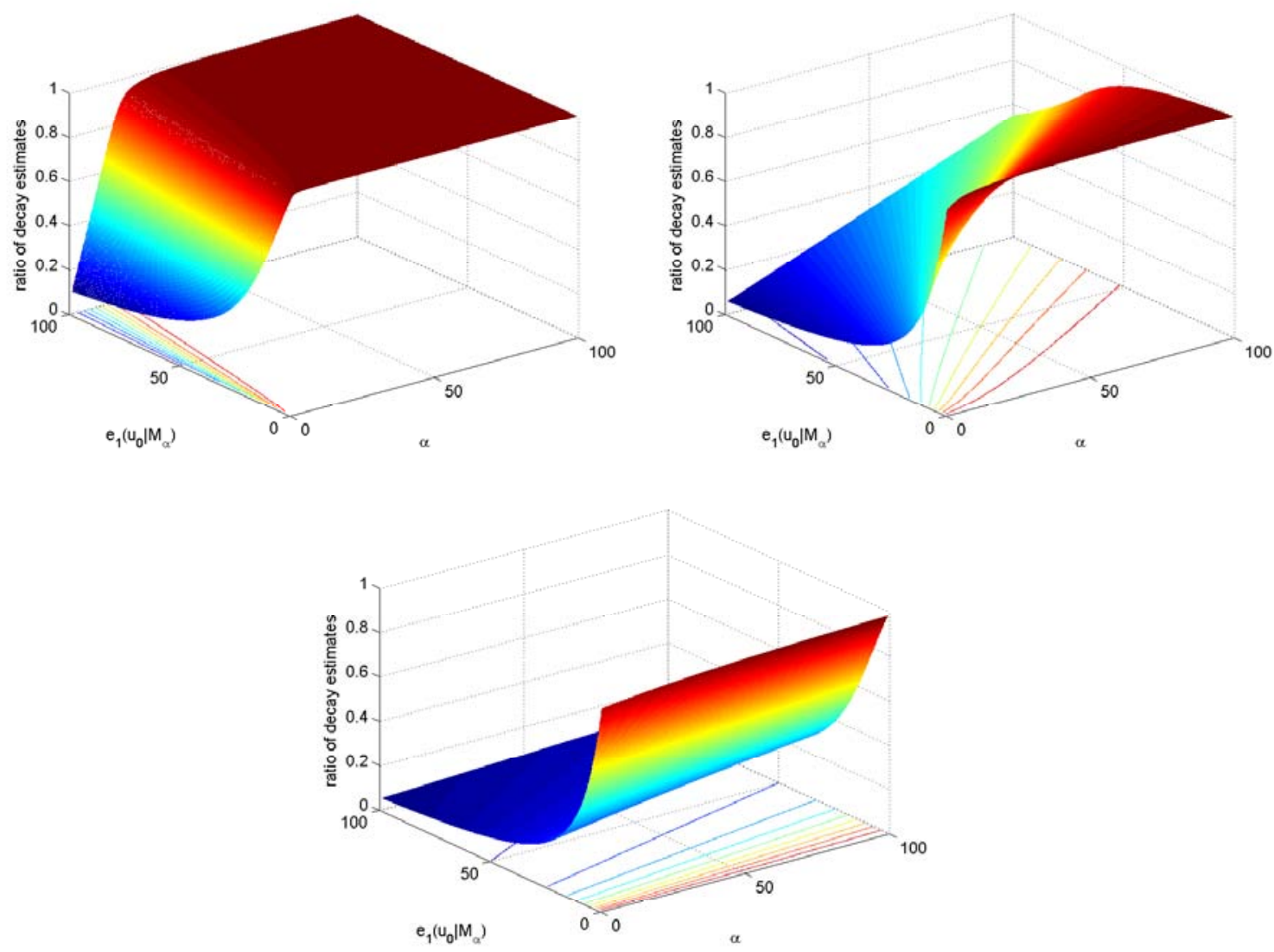

Figure 2: $R(0.1), R(1)$, and $R(10)$ for $n=1$ and $k=1$.

Therefore, our new decay estimate does not improve the decay rate at $\infty$ that one may expect from centering and normalizing the Gaussian approximation (at least in $L^{1}$ and in weighted $L^{2}$-spaces). However, it does improve at the level of constants of decay, i.e., at the level of the ratio of the improved decay estimate (2.23) to the original estimate (2.13) w.r.t. to the time $t>0$. We define the function $R: \mathbb{R}^{+} \longrightarrow \mathbb{R}^{+}$describing this ratio by

$$
R(t):=\frac{\frac{1}{k t}\left(\frac{\alpha}{2}-\frac{n}{2} E_{\min }(t)\right)}{\frac{\frac{\alpha}{n}}{\frac{\alpha}{n}+k t} e_{1}\left(u_{0} \mid M_{\frac{\alpha}{n}}\left(\cdot-x_{0}\right)\right)} .
$$

Using the approximation (2.25) of $E_{\min }(t)$ for large times $t$ gives

$$
\begin{aligned}
R(t) & =\frac{\frac{\frac{\alpha}{2}-\frac{n}{2} E_{\min }^{\infty}}{E_{\min }^{\infty}+k t}}{\frac{\frac{\alpha}{n}}{\frac{\alpha}{n}+k t} e_{1}\left(u_{0} \mid M_{\frac{\alpha}{n}}\left(\cdot-x_{0}\right)\right)}+\mathcal{O}\left(t^{-2}\right) \\
& =\frac{\frac{\alpha}{2}-\frac{n}{2} E_{\min }^{\infty}}{\frac{\alpha}{n} e_{1}\left(u_{0} \mid M_{\frac{\alpha}{n}}\left(\cdot-x_{0}\right)\right)} \cdot \frac{\frac{\alpha}{n}+k t}{E_{\text {min }}^{\infty}+k t}+\mathcal{O}\left(t^{-2}\right), \quad \forall t \gg 1 .
\end{aligned}
$$

We find that the ratio of the estimates is decreasing w.r.t. large times $t \gg 1$. Since the estimates coincide initially, the improvement of the decay rate becomes better for large 
times $t$ and in the limit $t \rightarrow \infty$ the ratio converges with rate $\left(E_{m i n}^{\infty}+k t\right)^{-1}$ to

$$
R(\infty)=\frac{\frac{\alpha}{2}-\frac{n}{2} E_{\min }^{\infty}}{\frac{\alpha}{n} e_{1}\left(u_{0} \mid M_{\frac{\alpha}{n}}\left(\cdot-x_{0}\right)\right)}
$$

Since $E_{\text {min }}^{\infty}=\frac{\alpha}{n} \exp \left[-\frac{2}{n} e_{1}\left(u_{0} \mid M_{\frac{\alpha}{n}}\left(\cdot-x_{0}\right)\right]\right.$ we find that the ratio $R(t)$ converges to

$$
\frac{1-\exp \left[-\frac{2}{n} e_{1}\left(u_{0} \mid M_{\frac{\alpha}{n}}\left(\cdot-x_{0}\right)\right)\right]}{\frac{2}{n} e_{1}\left(u_{0} \mid M_{\frac{\alpha}{n}}\right)}
$$

as the time $t$ goes to infinity. This limit is monotonically decreasing for increasing logarithmic entropies $e_{1}\left(u_{0} \mid M_{\underline{\alpha}}\left(\cdot-x_{0}\right)\right)>0$. We finally point out that the function $E_{\min }(t)$ as defined by (2.18) only depends on the variance of $u_{0}$ and on its relative entropy w.r.t. the Gaussian $M_{\frac{\alpha}{n}}\left(\cdot-x_{0}\right)$. Figure 2 shows the ratio function $R(t)$ for different times as a function of $\alpha:=\int\left|x-x_{0}\right|^{2} u_{0}(x) d x$ and the relative entropy $e_{1}\left(u_{0} \mid M_{\frac{\alpha}{n}}\left(\cdot-x_{0}\right)\right)$.

Example 2.5 We consider the initial function $u_{0}$ on $\mathbb{R}$ defined by

$$
u_{0}(x):= \begin{cases}\frac{1}{2\left(x_{2}-x_{1}\right)}, & x_{1} \leq|x| \leq x_{2} \\ 0, & \text { otherwise }\end{cases}
$$

where $x_{2}>x_{1}>0$. It is an even probability density with second moment $\alpha=\frac{1}{3}\left(x_{1}^{2}+\right.$ $\left.x_{1} x_{2}+x_{2}^{2}\right)$ and relative entropy $e_{1}\left(u_{0} \mid M_{\alpha}\right)=-\ln \left(2\left(x_{2}-x_{1}\right)\right)+\frac{1}{2} \ln (2 \pi \alpha)+\frac{1}{2}$. Choosing $x_{1}=1$ and $x_{2}=1.1$, we get $\alpha \approx 1.1033, e_{1}\left(u_{0} \mid M_{\alpha}\right) \approx 3.0775$ and $E_{\min }^{\infty} \approx 0.0023$. The limit (2.27) of the ratio of the improved decay estimate to the original one is approximately 0.1621. Figure 3 shows a comparison of the two decay estimates: the original (2.13) and the improved (2.23).
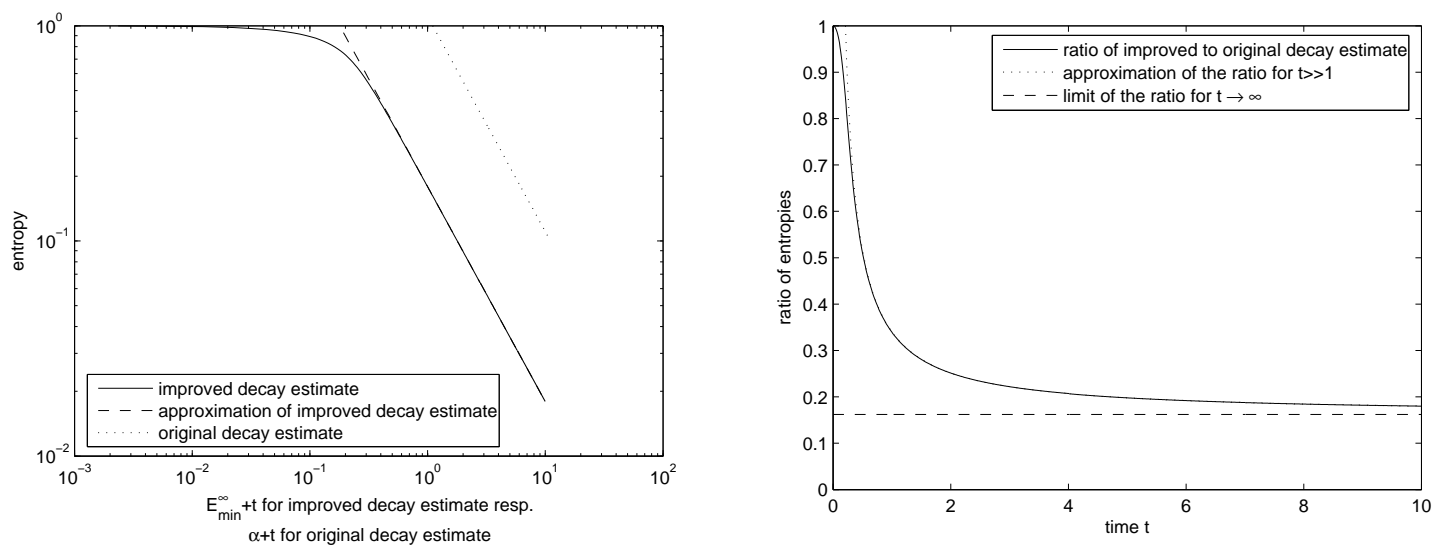

Figure 3: Left: Logarithmic plot of the original decay estimate (2.13), the improved decay estimate (2.23), and the approximation (2.25) of the improved decay estimate for $t \gg 1$ divided by $e_{1}\left(u_{0} \mid M_{\alpha}\right)$. The original decay estimate is plotted against $\alpha+t$ and the improved decay estimate as well as its approximation against $E_{m i n}^{\infty}+t$ in order to verify its asymptotic behaviors $(\alpha+t)^{-1}$ and $\left(E_{\text {min }}^{\infty}+t\right)^{-1}$, resp. Right: Ratio of the original (2.13) to the improved decay estimate (2.23), the approximation (2.26) of the ratio for $t \gg 1$, and the limit (2.27) of this approximation for $t \rightarrow \infty$. 
Remark 2.6 ( $L^{1}$-decay) Using the well known Csizár-Kullback inequality for probability densities [Cs63, Ku59]:

$$
\left\|\rho_{1}-\rho_{2}\right\|_{L^{1}\left(\mathbb{R}^{n}\right)}^{2} \leq 2 e_{1}\left(\rho_{1} \mid \rho_{2}\right),
$$

the above decay estimates imply analogous results in $L^{1}$.

Remark 2.7 (More General Parabolic Equations) In the spirit of [To96], we can extend Theorem 2.4 to derive improved decay estimates for uniformly parabolic equations of the form

$$
\frac{\partial u}{\partial t}=\frac{k}{2} \operatorname{div}([\mathbb{I}+A(x, t)] \nabla u) .
$$

Here, II is the identity matrix on $\mathbb{R}^{n}$. The symmetric positive perturbation $A$ as well as its first spatial derivative are supposed to decay like $(1+t)^{-\beta}$ for some $0<\beta<1$ (cf. [To96, K106] for details).

Remark 2.8 (Fokker-Planck equations) Using the time dependent rescaling

$$
u(\xi, \tau)=R(\tau)^{-n} v\left(\frac{\xi}{R(\tau)}, \ln R(\tau)\right)
$$

with $R(\tau)=\sqrt{k \tau+1}$ transforms the heat equation (2.1) into the Fokker-Planck equation

$$
\frac{\partial v}{\partial t}=\operatorname{div}(\nabla v+x v), \quad x \in \mathbb{R}^{n}, t>0 .
$$

Hence, the new decay estimate from Theorem 2.4 immediately translates into an improved decay estimate for the solution of the Fokker-Planck equation towards its unique normalized steady state $v_{\infty}(x)=e^{-|x|^{2} / 2}$ (cf. [K106] for details).

\section{The heat equation and non-isotropic Gaussians}

Up to now we have considered decay estimates for the heat equation w.r.t. isotropic Gaussians of the shape (2.2). Various convergence rates for the heat equation to more universal Gaussian densities in $\mathbb{R}^{n}$, namely Gaussians with an arbitrary covariance matrix, were found in [GJT02]. Motivated by these results we shall now generalize our convergence rates in logarithmic entropy of Section 2 to solutions for the heat equation with respect to general non-isotropic Gaussians:

$$
M_{\Sigma}(x):=(2 \pi)^{-n / 2}(\operatorname{det} \Sigma)^{-1 / 2} \exp \left(-\frac{1}{2} \Sigma^{-1} x \cdot x\right), \quad x \in \mathbb{R}^{n}
$$

where the covariance matrix $\Sigma \in \mathbb{R}^{n \times n}$ is symmetric and positive definite.

Given an initial probability density $u_{0}$ on $\mathbb{R}^{n}$ with finite second moments and an arbitrary $x_{0} \in \mathbb{R}^{n}$, we define the positive definite matrix $K(t)=\left(K_{i j}(t)\right)_{i, j=1, \ldots, n}$ for all times $t \geq 0$ by

$$
K_{i j}(t):=\int_{\mathbb{R}^{n}}\left(x-x_{0}\right)_{i}\left(x-x_{0}\right)_{j} u(x, t) d x, \quad i, j=1, \ldots, n,
$$


where $u$ is the solution of the heat equation (2.1). It is a simple matter to check that the evolution of the second moments of the solution is linear in time, more precisely, $K(t)=K(0)+k t \mathbb{I}$ for all $t \geq 0$.

Let us remark that the $n$-dimensional Gaussian density with first moment $x_{0}$ and covariance matrix $K(t), M_{K(t)}\left(x-x_{0}\right)$ is a solution itself to the heat equation (2.1). Since the heat equation is invariant under rotation of the coordinate system, we shall assume w.r.o.g. that $K(0)$ is diagonal. Since $K(t)=K(0)+k t \mathbb{I}$ is then diagonal for all $t \geq 0$. Hence, $M_{K(t)}\left(x-x_{0}\right)$ is a tensor product of $1 \mathrm{D}$ Gaussians, each of which satisfies the 1D heat equation.

\subsection{Decay in relative entropy}

The linear growth in time of the covariance matrix of general solutions motivates to consider its entropy behavior w.r.t. Gaussians with a covariance matrix of the form $\mathbb{E}+k t \mathbb{I}$, where $\mathbb{E} \in \mathbb{R}^{n \times n}$ is an arbitrary positive definite and symmetric matrix. We start with a lemma that is similar to [To96, Lemma 1]:

Lemma 3.1 (Finite Relative Fisher Information) Let the initial value $u_{0} \in C\left(\mathbb{R}^{n}\right) \cap$ $W_{\text {loc }}^{1,2}\left(\mathbb{R}^{n}\right) \cap L_{+}^{1}\left(\mathbb{R}^{n}\right)$ be a probability density on $\mathbb{R}^{n}$ with finite second moments. Then there is a constant $C>0$ such that the solution $u$ of the IVP (2.1) satisfies

$$
I\left(u(t) \mid u_{\infty}(t)\right)<C, \quad \forall t \in\left[t_{1}, t_{2}\right], 0<t_{1}<t_{2}<\infty
$$

with $u_{\infty}(x, t):=M_{\mathbb{E}+k t \mathbb{I}}\left(x-x_{0}\right)$ for an arbitrary $x_{0} \in \mathbb{R}^{n}$ and an arbitrary symmetric and positive definite matrix $\mathbb{E} \in \mathbb{R}^{n \times n}$. Moreover,

$$
\begin{gathered}
\lim _{\left|x_{j}\right| \rightarrow \infty} \frac{\partial u(t)}{\partial x_{j}}\left(\ln \frac{u(t)}{u_{\infty}(t)}+1\right)=0, \\
\lim _{\left|x_{j}\right| \rightarrow \infty} \frac{\partial u_{\infty}(t)}{\partial x_{j}} \frac{u(t)}{u_{\infty}(t)}=0
\end{gathered}
$$

for all $t>0$ and $j=1, \ldots, n$.

Proof. We calculate for all $t>0$

$$
\frac{\partial}{\partial x_{i}} \frac{u}{u_{\infty}}=\frac{1}{u_{\infty}} \frac{\partial u}{\partial x_{i}}+\frac{u}{u_{\infty}}\left((\mathbb{E}+k t \mathbb{I})^{-1} x\right)_{i}
$$

and find

$$
\left|\nabla \frac{u}{u_{\infty}}\right|^{2}=\frac{1}{\left(u_{\infty}\right)^{2}}|\nabla u|^{2}+\frac{u^{2}}{\left(u_{\infty}\right)^{2}}\left|(\mathbb{E}+k t \mathbb{I})^{-1} x\right|^{2}+\frac{u}{\left(u_{\infty}\right)^{2}} 2\left((\mathbb{E}+k t \mathbb{I})^{-1} x\right) \cdot \nabla u .
$$

This leads to

$$
I\left(u(t) \mid u_{\infty}(t)\right)=\int_{\mathbb{R}^{n}} \frac{1}{u}|\nabla u|^{2} d x+\int_{\mathbb{R}^{n}}\left|(\mathbb{E}+k t \mathbb{I})^{-1} x\right|^{2} u d x+2 \int_{\mathbb{R}^{n}}(\mathbb{E}+k t \mathbb{I})^{-1} x \cdot \nabla u d x,
$$

and the last term equals $-2 \operatorname{tr}(\mathbb{E}+k t \mathbb{I})^{-1}$. 
It is proved in [To96] that the first integral in the expression (3.6) is bounded for an initial function $u_{0} \in C\left(\mathbb{R}^{n}\right) \cap W_{l o c}^{1,2}\left(\mathbb{R}^{n}\right)$ :

$$
\int_{\mathbb{R}^{n}} \frac{1}{u}|\nabla u|^{2} d x \leq \int_{\mathbb{R}^{n}} \frac{1}{M_{k t}}\left|\nabla M_{k t}\right|^{2} d x=\frac{n}{k t} \leq \frac{n}{k t_{1}}, \quad t \geq t_{1} .
$$

Hence,

$$
I\left(u(t) \mid u_{\infty}(t)\right)=\int_{\mathbb{R}^{n}} \frac{1}{u}|\nabla u|^{2} d x+\int_{\mathbb{R}^{n}}\left|(\mathbb{E}+k t \mathbb{I})^{-1} x\right|^{2} u d x-2 \operatorname{tr}(\mathbb{E}+k t \mathbb{I})^{-1}<C,
$$

for all $t \geq t_{1}>0$. In [To96] Toscani also showed

$$
\lim _{\left|x_{j}\right| \rightarrow \infty} \frac{\partial u}{\partial x_{j}}(\ln u+1)=0 .
$$

Since $u$ and $\frac{\partial u}{\partial x_{j}}$ are smooth, fast-decaying at infinity functions and have finite second order moments, we deduce

$$
-\lim _{\left|x_{j}\right| \rightarrow \infty} \frac{\partial u}{\partial x_{j}} \ln u_{\infty}=\frac{1}{2} \lim _{\left|x_{j}\right| \rightarrow \infty} \frac{\partial u}{\partial x_{j}}\left[(\mathbb{E}+k t \mathbb{I})^{-1} x \cdot x+C(t)\right]=0
$$

and

$$
\lim _{\left|x_{j}\right| \rightarrow \infty} \frac{\partial u_{\infty}}{\partial x_{j}} \frac{u}{u_{\infty}}=-\lim _{\left|x_{j}\right| \rightarrow \infty}\left((\mathbb{E}+k t \mathbb{I})^{-1} x\right)_{j} u=0,
$$

concluding the proof.

This lemma leads to the proof of a decay rate in relative entropy for the solution of the heat equation w.r.t. general Gaussians.

Theorem 3.2 (Basic Decay Estimate) Let the initial value $u_{0} \in C\left(\mathbb{R}^{n}\right) \cap W_{\text {loc }}^{1,2}\left(\mathbb{R}^{n}\right) \cap$ $L_{+}^{1}\left(\mathbb{R}^{n}\right)$ be a probability density on $\mathbb{R}^{n}$ with finite second moment and entropy. Then the relative entropy of the solution $u$ to the IVP (2.1) w.r.t. $u_{\infty}(x, t):=M_{\mathbb{E}+k t \mathbb{I}}\left(x-x_{0}\right)$ with an arbitrary $x_{0} \in \mathbb{R}^{n}$ and an arbitrary symmetric and positive definite matrix $\mathbb{E} \in \mathbb{R}^{n \times n}$ converges to zero as $t \rightarrow \infty$. More precisely,

$$
e_{1}\left(u(t) \mid u_{\infty}(t)\right) \leq \frac{\rho(\mathbb{E})}{\rho(\mathbb{E})+n k t} e_{1}\left(u_{0} \mid u_{\infty}(0)\right), \quad \forall t \geq 0,
$$

where $\rho(\mathbb{E})$ denotes the spectral radius of $\mathbb{E}$.

Proof. Following the proof of Theorem 2.1 we obtain for all $t>0$

$$
\begin{aligned}
\frac{d}{d t} e_{1}\left(u(t) \mid u_{\infty}(t)\right) & =\int_{\mathbb{R}^{n}} \frac{\partial u}{\partial t}\left[\ln \left(\frac{u}{u_{\infty}}\right)+1\right] d x-\int_{\mathbb{R}^{n}} \frac{\partial u_{\infty}}{\partial t} \frac{u}{u_{\infty}} d x \\
& =\frac{k}{2} \int_{\mathbb{R}^{n}} \triangle u\left[\ln \left(\frac{u}{u_{\infty}}\right)+1\right] d x-\frac{k}{2} \int_{\mathbb{R}^{n}} \triangle u_{\infty} \frac{u}{u_{\infty}} d x \\
& =-\frac{k}{2} \int_{\mathbb{R}^{n}} \frac{u_{\infty}}{u} \nabla u \cdot \nabla\left(\frac{u}{u_{\infty}}\right) d x+\frac{k}{2} \int_{\mathbb{R}^{n}} \nabla u_{\infty} \cdot \nabla \frac{u}{u_{\infty}} d x \\
& =-\frac{k}{2} I\left(u(t) \mid u_{\infty}(t)\right),
\end{aligned}
$$


where $I\left(u(t) \mid u_{\infty}(t)\right)$ is the relative Fisher information (2.5) of $u$ w.r.t. $u_{\infty}$. We conclude that the relative entropy $e_{1}\left(u(t) \mid u_{\infty}(t)\right)$ is monotonic decreasing w.r.t. time. In the above integrations by parts, the boundary terms disappear due to Lemma 3.1.

Next we shall apply a LSI for the measure $u_{\infty}(t)$. To this end we use $\rho(\mathbb{E}) \mathbb{I} \geq \mathbb{E}>0$ and hence

$$
(\mathbb{E}+k t \mathbb{I})^{-1} \geq(\rho(\mathbb{E})+k t)^{-1} \mathbb{I}, \quad t \geq 0 .
$$

Since

$$
\operatorname{Hess}_{x}\left[-\ln u_{\infty}(x, t)\right]=\operatorname{Hess}_{x}\left[-\ln M_{\mathbb{E}+k t \mathbb{I}}\left(x-x_{0}\right)\right]=(\mathbb{E}+k t \mathbb{I})^{-1},
$$

(3.10) shows that $u_{\infty}(t)$ is uniformly log-concave with lower bound $(\rho(\mathbb{E})+k t)^{-1}$. Thus, (3.10) is a Bakry-Emery condition for the probability density $u_{\infty}(t)$, cf. [BE84, BE85, AMTU01]. Hence, $u_{\infty}(t)$ satisfies the LSI

$$
e_{1}\left(\rho \mid u_{\infty}(t)\right) \leq \frac{\rho(\mathbb{E})+k t}{2} I\left(\rho \mid u_{\infty}(t)\right)
$$

$\forall \rho \in L_{+}^{1}\left(\mathbb{R}^{n}\right)$ with $\int \rho d x=1$. Combining (3.9) and (3.11) yields

$$
\frac{d}{d t} e_{1}\left(u(t) \mid u_{\infty}(t)\right) \leq-\frac{k}{\rho(\mathbb{E})+k t} e_{1}\left(u(t) \mid u_{\infty}(t)\right), \quad t>0
$$

and Gronwall's lemma implies the decay estimate (3.8).

\subsection{Improved decay estimate in relative entropy}

Following the strategy of Section 2 we shall improve the decay estimate of Theorem 3.2 for the solution of the heat equation. In a first step we identify, for each fixed $t \geq 0$, the optimal non-isotropic Gaussian in the same sense as in the case of standard Gaussians. This shall yield by a minimization method an improvement of the convergence rate in relative entropy w.r.t. general Gaussians.

We consider an initial probability density $u_{0}$ on $\mathbb{R}^{n}$ with its center of mass at $x_{0} \in \mathbb{R}^{n}$, i.e. $\int\left(x-x_{0}\right) u_{0} d x=0$, and with $\int\left|x-x_{0}\right|^{2} u_{0} d x<\infty$. For all $t \geq 0$ the covariance matrix $K(t) \in \mathbb{R}^{n \times n}$ of the solution $u(t)$ to the heat equation is defined by

$$
K_{i j}(t):=\int_{\mathbb{R}^{n}}\left(x-x_{0}\right)_{i}\left(x-x_{0}\right)_{j} u(x, t) d x, \quad i, j=1, \ldots, n .
$$

As in previous section, we know that $K(t)=K(0)+k t \mathbb{I}$.

Now we want to find the general Gaussian $u_{\infty}(x, t):=M_{\overline{\mathbb{E}}(t)}\left(x-\bar{x}_{0}\right)$ that minimizes (for each fixed $t \geq 0)$ the relative entropy $e_{1}\left(u(t) \mid u_{\infty}(t)\right)$. The optimal first moment $\bar{x}_{0} \in \mathbb{R}^{n}$ of $u_{\infty}$ and the optimal positive definite matrix $\overline{\mathbb{E}}(t) \in \mathbb{R}^{n \times n}$ are such that the $0^{\text {th }}$ and $1^{\text {st }}$ moments of $u(t)$ and $u_{\infty}(t)$, as well as their covariance matrices coincide:

Theorem 3.3 (Optimal Non-isotropic Gaussian) Let the initial value $u_{0}$ be a probability density on $\mathbb{R}^{n}$ with finite second moment and entropy. Then, for each fixed time $t \geq 0, e_{1}\left(u(t) \mid M_{K(0)+k t \mathbb{I}}\left(\cdot-x_{0}\right)\right)$ is the smallest relative entropy of the solution $u$ of the $\operatorname{IVP}(2.1)$ w.r.t. all general Gaussians $M_{\tilde{\mathbb{E}}(t)}\left(x-\tilde{x}_{0}\right)$ with an arbitrary $\tilde{x}_{0} \in \mathbb{R}^{n}$ and an arbitrary positive definite matrix $\tilde{\mathbb{E}}(t) \in \mathbb{R}^{n \times n}$. 
Proof. The relative entropy reads

$$
\begin{aligned}
& e_{1}\left(u(t) \mid M_{\tilde{\mathbb{E}}(t)}\left(\cdot-\tilde{x}_{0}\right)\right) \\
& =\int_{\mathbb{R}^{n}} u \ln u d x+\frac{n}{2} \ln \left(2 \pi[\operatorname{det} \tilde{\mathbb{E}}(t)]^{1 / n}\right)+\frac{1}{2} \int_{\mathbb{R}^{n}} \tilde{\mathbb{E}}(t)^{-1}\left(x-\tilde{x}_{0}\right) \cdot\left(x-\tilde{x}_{0}\right) u d x .
\end{aligned}
$$

$f\left(\tilde{x}_{0}\right)$, defined as the third term on the right-hand side of this equation is minimal w.r.t. $\tilde{x}_{0} \in \mathbb{R}^{n}$ if and only if

$$
\nabla f\left(\tilde{x}_{0}\right)=-\int_{\mathbb{R}^{n}} \tilde{\mathbb{E}}(t)^{-1}\left(x-\tilde{x}_{0}\right) u(x, t) d x=0 .
$$

Since the matrix $\tilde{\mathbb{E}}(t)$ is regular, this condition is equivalent to

$$
\int_{\mathbb{R}^{n}}\left(x-\tilde{x}_{0}\right) u(x, t) d x=0 .
$$

Since $u(x, t)$ conserves the center of mass (cf. (2.10)) we conclude: The relative entropy $e_{1}\left(u(t) \mid M_{\tilde{\mathbb{E}}(t)}\left(\cdot-\tilde{x}_{0}\right)\right)$ is minimal w.r.t. $\tilde{x}_{0} \in \mathbb{R}^{n}$ iff the first moments of $u$ and $M_{\tilde{\mathbb{E}}(t)}\left(x-\tilde{x}_{0}\right)$ coincide, i.e. iff $x_{0}=\tilde{x}_{0}$.

To determine the positive definite matrix $\overline{\mathbb{E}} \in \mathbb{R}^{n \times n}$ minimizing, for each fixed $t \geq 0$, the relative entropy (3.13), we have to minimize

$$
\ln (\operatorname{det} \tilde{\mathbb{E}})+\operatorname{tr}\left(\tilde{\mathbb{E}}^{-1} K\right)
$$

w.r.t. all positive definite matrices $\tilde{\mathbb{E}}=\tilde{\mathbb{E}}(t) . K=K(0)+k t \mathbb{I}$ denotes here the covariance matrix of $u(t)$. To simplify the computation we put $\mathbb{F}:=\sqrt{K} \tilde{\mathbb{E}}^{-1} \sqrt{K} \geq 0$. Using the cyclicity of the trace we now have to minimize

$$
-\ln (\operatorname{det} \mathbb{F})+\operatorname{tr}(\mathbb{F})=\sum_{j=1}^{n}\left(\lambda_{j}-\ln \lambda_{j}\right)
$$

w.r.t. all positive definite matrices $\mathbb{F}$, with $\lambda_{j}$ denoting its eigenvalues. Clearly, the unique minimum is attained at $\mathbb{F}=\mathbb{I}$, or equivalently, at $\overline{\mathbb{E}}=K$.

With this knowledge of the optimal Gaussian we are able to improve our decay estimate (3.8) for the solution of the heat equation w.r.t. general Gaussians, using a method similar to the one of Section 2. Theorem 3.2 yields for the solution $u(t)$ of the heat equation (2.1) the decay estimate

$$
e_{1}\left(u(t) \mid M_{\mathbb{E}+k t \mathbb{I}}\left(\cdot-\tilde{x}_{0}\right)\right) \leq \frac{\rho(\mathbb{E})}{\rho(\mathbb{E})+n k t} e_{1}\left(u_{0} \mid M_{\mathbb{E}}\left(\cdot-\tilde{x}_{0}\right)\right)
$$

for an arbitrary $\tilde{x}_{0} \in \mathbb{R}^{n}$ and an arbitrary positive definite matrix $\mathbb{E} \in \mathbb{R}^{n \times n}$. We estimate like in (2.16):

$$
\begin{aligned}
e_{1}\left(u(t) \mid M_{K(0)+k t \mathbb{I}}\left(\cdot-x_{0}\right)\right) & =\min _{\substack{\mathbb{E}>0 \\
\tilde{x}_{0} \in \mathbb{R}^{n}}} e_{1}\left(u(t) \mid M_{\mathbb{E}+k t \mathbb{I}}\left(\cdot-\tilde{x}_{0}\right)\right) \\
& \leq \inf _{\substack{\mathbb{E}>0 \\
\tilde{x}_{0} \in \mathbb{R}^{n}}} \frac{\rho(\mathbb{E})}{\rho(\mathbb{E})+n k t} e_{1}\left(u_{0} \mid M_{\mathbb{E}}\left(\cdot-\tilde{x}_{0}\right)\right) \\
& =\inf _{\mathbb{E}>0} \frac{\rho(\mathbb{E})}{\rho(\mathbb{E})+n k t} e_{1}\left(u_{0} \mid M_{\mathbb{E}}\left(\cdot-x_{0}\right)\right) .
\end{aligned}
$$


In the case $u_{0}(x)=M_{K(0)}\left(x-x_{0}\right)$ we find that $e_{1}\left(u(t) \mid M_{K(0)+k t \mathbb{I}}\left(\cdot-x_{0}\right)\right)=0$ for all times $t \geq 0$ and it holds equality in (3.15). Hence, we obtain the minimum of the right-hand side of formula (3.16) for $\mathbb{E}=K(0)$, i.e. (3.15) is already optimal.

In the case $u_{0}(x) \neq M_{K(0)}\left(x-x_{0}\right)$ we have to minimize the function $f$, defined for each $t \geq 0$ on the cone of positive definite matrices:

$$
\begin{aligned}
f(\mathbb{E}, t) & :=\frac{\rho(\mathbb{E})}{\rho(\mathbb{E})+n k t} e_{1}\left(u_{0} \mid M_{\mathbb{E}}\left(\cdot-x_{0}\right)\right) \\
& =\frac{1}{2} \frac{\rho(\mathbb{E})}{\rho(\mathbb{E})+n k t}\left(2 \int_{\mathbb{R}^{n}} u_{0} \ln u_{0} d x+n \ln (2 \pi)+\ln (\operatorname{det} \mathbb{E})+\operatorname{tr}\left(\mathbb{E}^{-1} K(0)\right)\right) \geq 0
\end{aligned}
$$

w.r.t. $\mathbb{E} \geq 0$. This function has the following features.

Lemma 3.4 (Computation of $\mathbb{E}_{\text {min }}(t)$ ) For each fixed $t \geq 0$ the function $f(\mathbb{E}, t)$ has w.r.t. to all symmetric and positive definite matrices $\mathbb{E} \in \mathbb{R}^{n \times n}$ a unique minimum at $\mathbb{E}_{\min }(t) \in \mathbb{R}^{n \times n}$ with the following properties:

a) $\mathbb{E}_{\text {min }}=\mathbb{E}_{\text {min }}(t)$ satisfies

$$
\mathbb{E}_{\text {min }}=\min \left(K(0), \rho_{\text {min }} \mathbb{I}\right),
$$

where $\rho_{\text {min }}=\rho\left(\mathbb{E}_{\text {min }}\right) \leq \rho(K(0))$ is its spectral radius.

b) $\mathbb{E}_{\min }(0)=K(0)$.

c) $\mathbb{E}_{\text {min }}(t)$ is monotonic decreasing w.r.t. $t \geq 0$, i.e. $\mathbb{E}_{\min }\left(t_{2}\right) \leq \mathbb{E}_{\min }\left(t_{1}\right)$ for $0 \leq t_{1}<t_{2}$ in the sense of positive definite matrices. In particular,

$$
0<\mathbb{E}_{\text {min }}^{\infty} \leq \mathbb{E}_{\text {min }}(t) \leq K(0), \quad \forall t \geq 0 .
$$

Proof. To simplify the notation we put $K=K(0)$. W.r.o.g. we shall assume $K=$ $\operatorname{diag}\left(k_{1}, \ldots, k_{n}\right)$ with $0<k_{1} \leq k_{2} \leq \ldots \leq k_{n}$. Indeed, if the minimum of $f_{K}(., t)$ is attained at $\mathbb{E}=\mathbb{E}_{\text {min }}$, the minimum of $f_{\tilde{K}}(., t)$ with $\tilde{K}:=S K S^{-1}$ and $S$ orthogonal is attained at $\tilde{\mathbb{E}}_{\text {min }}:=S \mathbb{E}_{\text {min }} S^{-1}$. This follows from $\rho\left(\mathbb{E}_{\text {min }}\right)=\rho\left(\tilde{\mathbb{E}}_{\text {min }}\right), \operatorname{det}\left(\mathbb{E}_{\text {min }}\right)=\operatorname{det}\left(\tilde{\mathbb{E}}_{\text {min }}\right)$, $\operatorname{tr}\left(\mathbb{E}_{\text {min }}^{-1} K\right)=\operatorname{tr}\left(\tilde{\mathbb{E}}_{\text {min }}^{-1} \tilde{K}\right)$, and hence $f_{K}\left(\mathbb{E}_{\text {min }}, t\right)=f_{\tilde{K}}\left(\tilde{\mathbb{E}}_{\text {min }}, t\right)$.

We shall now minimize $f(\mathbb{E}, t)$ w.r.t. $\mathbb{E}$ positive definite and symmetric in three steps, to show that we can reduce to minimize a function of the spectrum of $\mathbb{E}$ :

$$
\begin{aligned}
\min _{\mathbb{E}>0} f(\mathbb{E}, t) & =\min _{\rho>0} f_{1}(\rho, t)\left[\min _{\substack{\mathbb{E}>0 \\
\rho(\mathbb{E})=\rho}} f_{2}(\mathbb{E})\right] \\
& =\min _{\rho>0} f_{1}(\rho, t)\left\{\min _{\substack{\sigma(\mathbb{E}) \subset \mathbb{R}^{+} \\
\rho(\mathbb{E})=\rho \text { fixed }}}\left[\beta+\ln (\operatorname{det} \mathbb{E})+\min _{\substack{\mathbb{E}>0 \\
\sigma(\mathbb{E}) \subset \mathbb{R}^{+} \text {fixed }}} \operatorname{tr}\left(\mathbb{E}^{-1} K\right)\right]\right\},
\end{aligned}
$$

with the scalar functions

$$
\begin{gathered}
f_{1}(\rho, t):=\frac{1}{2} \frac{\rho}{\rho+n k t}, \quad \rho>0 \\
f_{2}(\mathbb{E}):=\beta+\ln (\operatorname{det} \mathbb{E})+\operatorname{tr}\left(\mathbb{E}^{-1} K\right)=\beta+\sum_{j=1}^{n}\left(\ln e_{j}+\frac{k_{j}}{e_{j}}\right), \quad \mathbb{E}>0,
\end{gathered}
$$


with $\beta:=2 \int u_{0} \ln u_{0} d x+n \ln (2 \pi)$ and $\sigma(\mathbb{E})=\left\{0<e_{1} \leq \ldots \leq e_{n}=\rho\right\}$.

Step 1:

First we shall minimize $\operatorname{tr}\left(\mathbb{E}^{-1} K\right)$ over all symmetric matrices $\mathbb{E}$ having the fixed spectrum $\sigma(\mathbb{E})=\left\{0<e_{1} \leq \ldots \leq e_{n}=\rho\right\}$. Since $K$ is diagonal, the minimum of $\operatorname{tr}\left(\mathbb{E}^{-1} K\right)$ is attained at $\mathbb{E}_{3}=\operatorname{diag}\left(e_{1}, \ldots, e_{n}\right)$. Since the entries of $K$ are increasing, also the $e_{j}$ 's have to increase. This is a direct consequence of the following result (Theorem 1 of [Ri58]): For all real symmetric matrices $A, B$ it holds:

$$
\sum_{j=1}^{n} \lambda_{n-j+1}(A) \lambda_{j}(B) \leq \operatorname{tr}(A B) \leq \sum_{j=1}^{n} \lambda_{j}(A) \lambda_{j}(B),
$$

where the eigenvalues are labeled in increasing order. The right inequality is actually a special case of the von Neumann trace inequality [Mi75]. The left inequality now yields the assertion

$$
\operatorname{tr}\left(\mathbb{E}^{-1} K\right) \geq \sum_{j=1}^{n} \lambda_{n-j+1}\left(\mathbb{E}^{-1}\right) \lambda_{j}(K)=\sum_{j=1}^{n} \frac{k_{j}}{e_{j}}=\operatorname{tr}\left(\mathbb{E}_{3}^{-1} K\right) \quad \forall \mathbb{E}>0 \text { with } \sigma(\mathbb{E}) \text { fixed. }
$$

Step 2:

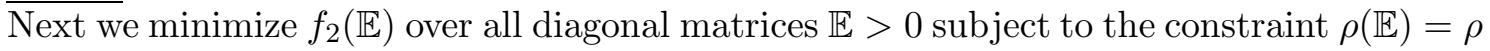
with $\sigma(\mathbb{E})=\left\{0<e_{1} \leq \ldots \leq e_{n}=\rho\right\}$. From this we can conclude that the unique minimum of $f_{2}(\mathbb{E})$ w.r.t. $\mathbb{E}>0$ and $\rho(\mathbb{E})=\rho$ (with $\rho$ fixed) is attained at $\mathbb{E}_{2}=\left(e_{21}, \ldots, e_{2 n}\right)$ with

$$
\begin{aligned}
& e_{2 j}=\min \left(k_{j}, \rho\right), \quad j \leq n-1, \\
& e_{2 n}=\rho .
\end{aligned}
$$

We first remark that each summand of (3.19) is a decreasing function of $e_{j}$ for $0<e_{j}<k_{j}$, increasing for $e_{j}>k_{j}$ achieving its minimum at $\bar{e}_{j}=k_{j}$. Now, the largest eigenvalue $e_{2 n}$ must be equal to $\rho$ by definition of the minimization set of matrices. Taking into account both facts we verify (3.20). Using (3.20) in (3.19), the minimum of $f_{2}(\mathbb{E})$ satisfies

$$
f_{3}(\rho):=\min _{\substack{\mathbb{E}>0 \\ \rho(\mathbb{E})=\rho}} f_{2}(\mathbb{E})=f_{2}\left(\mathbb{E}_{2}\right)=\beta+\ln \rho+\frac{k_{n}}{\rho}+\sum_{j=1}^{n-1} g_{j}(\rho), \quad \rho>0,
$$

with the $C^{1}\left(\mathbb{R}^{+}\right)$-functions, $j=1, \ldots, n-1$,

$$
g_{j}(\rho):= \begin{cases}\ln \rho+\frac{k_{j}}{\rho}, & \rho \leq k_{j} \\ \ln k_{j}+1, & \rho \geq k_{j}\end{cases}
$$

Step 3:

Next we minimize $f_{1}(\rho) f_{3}(\rho)$ w.r.t. $\rho>0$. This yields the following condition for $\rho_{\text {min }}$ :

$$
n k t\left(f_{3}+\rho f_{3}^{\prime}\right)=-\rho^{2} f_{3}^{\prime} .
$$

Here,

$$
f_{3}(\rho)+\rho f_{3}^{\prime}(\rho)=\beta+\ln \rho+1+\sum_{j=1}^{n-1}\left(g_{j}+\rho g_{j}^{\prime}\right)
$$


with

$$
g_{j}+\rho g_{j}^{\prime}= \begin{cases}\ln \rho+1, & \rho \leq k_{j}, \\ \ln k_{j}+1, & \rho \geq k_{j} .\end{cases}
$$

Hence, $f_{3}+\rho f_{3}^{\prime}$ is strictly monotonic increasing in $\rho>0$,

$$
\lim _{\rho \rightarrow 0+} f_{3}(\rho)+\rho f_{3}^{\prime}(\rho)=-\infty
$$

and

$$
f_{3}\left(k_{n}\right)+k_{n} f_{3}^{\prime}\left(k_{n}\right)=2 e\left(u_{0} \mid M_{K(0)}\left(.-x_{0}\right)\right) \geq 0 .
$$

On the other hand,

$$
-\rho^{2} f_{3}^{\prime}(\rho)=k_{n}-\rho+\sum_{j=1}^{n-1}\left(k_{j}-\rho\right) H\left(k_{j}-\rho\right)
$$

(with $H$ denoting the Heaviside function) is strictly monotonic decreasing in $\rho>0$, positive on $\left[0, k_{n}\right)$, and it has a unique zero at $\rho=k_{n}$. This implies that the equation (3.23) has a unique solution $\rho_{\min }$ with $0<\rho_{\min } \leq k_{n}$.

One easily checks that $\lim _{\rho \rightarrow 0+}\left(f_{1} f_{3}\right)^{\prime}(\rho)=-\infty$. Moreover, $f_{1}$ and $f_{3}$ are both strictly increasing on $\left[k_{n}, \infty\right)$. Hence, $\left(f_{1} f_{3}\right)(\rho)$ takes its unique minimum at $\rho=\rho_{\text {min }} \leq k_{n}$.

a) From $(3.20)$ we hence conclude $\mathbb{E}_{\min }=\min \left(K(0), \rho_{\min } \mathbb{I}\right)$.

b) $t=0$ implies $\rho_{\text {min }}=k_{n}$ and hence $\mathbb{E}_{\min }(0)=K(0)$.

c) For $u_{0} \neq M_{K(0)}\left(.-x_{0}\right)$, the monotonicity properties of both sides of (3.23) imply that $\rho_{\min }(t)$ is strictly decreasing in $t$, with

$$
0<\rho_{\min }^{\infty}<\rho_{\min }(t)<k_{n} \quad \forall t>0 .
$$

Here, $\rho_{\text {min }}^{\infty}$ is the unique minimum of $f_{3}+\rho f_{3}^{\prime}$ (cp. to the analogous situation in Lemma $2.3(\mathrm{~d})$ and in Figure 1). Hence, (3.20) implies that the matrix $\mathbb{E}_{\text {min }}(t)$ is decreasing w.r.t. $t$. In the case $u_{0}(x)=M_{K(0)}\left(x-x_{0}\right)$ we have $\mathbb{E}_{\min }(t)=K(0)$.

Remark 3.5 (Radial Symmetric Case) In the special case of a radially symmetric initial condition with covariance matrix $K(0)=\frac{\alpha}{n} \cdot \mathbb{I}$, the above Lemma 3.4 reduces to Lemma 2.3 with $\mathbb{E}_{\min }(t)=E_{\text {min }}(t) \cdot \mathbb{I}$ for $t \geq 0$. Then, the condition (3.23) is equivalent to

$$
\left(\int_{\mathbb{R}^{n}} u_{0}(x) \ln u_{0}(x) d x+\frac{n}{2} \ln \left(2 \pi E_{\min }(t)\right)+\frac{n}{2}\right) k t=\frac{\alpha}{2}-\frac{n}{2} E_{\min }(t) .
$$

Lemma 3.4 and Theorem 3.3 now directly yield an improved decay estimate in logarithmic entropy (compared to the result of Theorem 3.2): 
Theorem 3.6 (Improved Decay Estimate) Let $u_{0} \in C\left(\mathbb{R}^{n}\right) \cap W_{l o c}^{1,2}\left(\mathbb{R}^{n}\right) \cap L_{+}^{1}\left(\mathbb{R}^{n}\right)$ be a probability density on $\mathbb{R}^{n}$ with finite second moment and entropy. Then the solution $u$ of the IVP (2.1) satisfies

$$
e_{1}\left(u(t) \mid M_{K+k t \mathbb{I}(}\left(\cdot-x_{0}\right)\right) \leq \frac{\rho\left(\mathbb{E}_{\text {min }}(t)\right)}{\rho\left(\mathbb{E}_{\min }(t)\right)+n k t} e_{1}\left(u_{0} \mid M_{\mathbb{E}_{\text {min }}(t)}\left(\cdot-x_{0}\right)\right)=f\left(\mathbb{E}_{\text {min }}(t), t\right)
$$

with $\mathbb{E}_{\text {min }}(t)$ from Lemma 3.4 and Remark 3.5.

In particular, if $K(0)=\frac{\alpha}{n} \cdot \mathbb{I}$ we have $\mathbb{E}_{\min }(t)=E_{\min }(t) \cdot \mathbb{I}$ for $t \geq 0$, and Theorem 3.6 reduces to Theorem 2.4 for standard Gaussians.

Acknowledgment: AA was partially supported by the DFG under grant no. AR 277/3-3 and by the ESF in the project "Global and geometrical aspects of nonlinear partial differential equations". JAC acknowledges the support from DGI-MEC (Spain) FEDER-project MTM2005-08024 and 2005SGR00611. AA and JAC acknowledge partial support of the Acc. Integ. program HU2006-0025.

\section{References}

[An88] S. Angenent, Large time asymptotics for the porous media equation, Nonlinear diffusion equations and their equilibrium states, I (Berkeley, CA, 1986), Math. Sci. Res. Inst. Publ. 12, 21-34, Springer (1988).

[ACDD04] A. Arnold, J.A. Carrillo, L. Desvillettes, J. Dolbeault, A. Jüngel, C. Lederman, P.A. Markowich, G. Toscani, C. Villani, Entropies and Equilibria of Many-Particle Systems: An Essay on Recent Research, Monatshefte für Mathematik 142 (2004) 35-43.

[AMTU01] A. Arnold, P. Markowich, G. Toscani, A. Unterreiter, On convex Sobolev inequalities and the rate of convergence to equilibrium for Fokker-Plack type equations, Comm. PDE 26 (2001) 43-100.

[BE84] D. Bakry, M. Emery, Hypercontractivité de semi-groupes de diffusion, C. R. Acad. Sci. Paris Sér. I 15 (1984) 299-305.

[BE85] D. Bakry, M. Emery, Diffusions hypercontractivities, Sém. Prob. XIX, 1123 Lecture Notes in Math. Springer (1985) 177-206.

[Ba97] G.I. Barenblatt, Scaling, Self-similarity, and Intermediate Asymptotics, Cambridge University Press, 1996, reprinted 1997.

[Ca91] E.A. Carlen, Superadditivity of Fisher's Information and Logarithmic Sobolev Inequalities, J. Func. Anal. 101 (1991) 194-211.

[CC92] E.A. Carlen, M. Carvalho, Strict entropy production bounds and stability of the rate of convergence to equilibrium for the Boltzmann equation, J. Statist. Phys. 67 (1992) 575-608. 
[CDT07] J.A. Carrillo, M. DiFrancesco, and G. Toscani, Strict contractivity of the 2Wasserstein distance for the porous medium equation by mass-centering, Proc. Amer. Math. Soc. 135 (2007) 353-363.

[CJMTU01] J.A. Carrillo, A. Jüngel, P. Markowich, G. Toscani, A. Unterreiter, Entropy production methods for degenerate parabolic problems and generalized Sobolev inequalities, Monatshefte für Mathematik 133 (2001) 1-82.

[CLMT02] J.A. Carrillo, C. Lederman, P.A. Markowich, G. Toscani, Poincaré inequalities for lineraizations of very fast diffusion equations, Nonlinearity 15 (2002) 565-580.

[CT00] J.A. Carrillo, G. Toscani, Asymptotic $L^{1}$-decay of solutions of the porous medium equation to self-similarity, Indiana Univ. Math. J. 49 (2000) 113-142.

[CV03] J.A. Carrillo and J.L. Vázquez, Fine asymptotics for fast diffusion equations, Comm. PDE 28 (2003) 1023-1056.

[Ce82] C. Cercignani, H-Theorem and trend to equilibrium in the kinetic theory of gases, Arch. Mech. 34 (1982) 231-241.

[Cs63] I. Csiszár, Eine informationstheoretische Ungleichung und ihre Anwendung auf den Beweis von Markoffschen Ketten, Magyar Tud. Akad. Mat. Kutato Int. Kl. 8 (1963) 85-108.

[DM04] J. Denzler, R. McCann, Fast diffusion to Self-Similarity: Complete Spectrum, Long-time Asymtotics and Numerology, Arch. Rational Mech. Anal. 175 (2004) 301-342.

[DZ92] J. Duoandikoetxea, E. Zuazua, Moments, masses de Dirac et décomposition de fonctions, C. R. Acad. Sci. Paris Sér. I Math. 315 (1992) 693-698.

[Fi25] R. Fisher, Theory of statistical estimation, Math. Proc. Cambridge Philos. Soc. 22 (1925) 700-725.

[GJT02] T. Goudon, S. Junca, G. Toscani, Fourier-based metrics and Berry-Esseen like inequalities, Monatshefte für Mathematik 135 (2002) 115-136.

[Gr75] L. Gross, Logarithmic Sobolev inequalities, Amer. J. of Math. 97 (1975) 10611083.

[KM05] Y.-J. Kim, R.J. McCann, Sharp decay rates for the fastest conservative diffusions, C. R. Acad. Sci. Paris Ser. I Math. 341 (2005) 157-162.

[KM07] Y.-J. Kim, R.J. McCann, Potential theory and optimal convergence rates in fast nonlinear diffusion, J. Math. Pures Appl. 86 (2006) 42-67.

[K106] C. Klapproth, Entropy Methods for the Large-Time Behavior of Parabolic Equations, Master Thesis, Münster University, 2006.

[Ku59] S. Kullback, Information Theory and Statistics, John Wiley (1959). 
[MS06] R.J. McCann, D. Slepcev, Second-order asymptotics for the fast-diffusion equation, Int. Math. Res. Not. 24947 (2006) 1-22.

[Mi75] L. Mirsky, A trace inequality of John von Neumann, Monatshefte für Mathematik 79 (1975) 303-306.

[Ot01] F. Otto, The geometry of dissipative evolution equations: the porous medium equation, Comm. PDE 26 (2001) 101-174.

[Ri58] H. Richter, Zur Abschätzung von Matrizennormen, Math. Nachr. 18 (1958) 178-187.

[To96] G. Toscani, Kinetic approach to the asymptotic behaviour of the solution to diffusion equations, Rend. di Matematica 16 (1996) 329-346.

[To97] G. Toscani, Sur l'egalité logarithmique de Sobolev, C. R. Acad. Sci. Paris Math. 324 (1997) 689-694.

[UAMT00] A. Unterreiter, A. Arnold, P. Markowich, G. Toscani, On generalized Csiszár-Kullback inequalities, Monatshefte für Mathematik 131 (2000) 235-253.

[Va03] J.L. Vázquez, Asymptotic behaviour for the porous medium equation posed in the whole space, J. Evol. Equ. 3 (2003) 67-118.

[Va06] J.L. Vázquez. The Porous Medium Equation. Mathematical theory, Oxford Univ. Press, (2007).

[WB98] T. P. Witelski, A. J. Bernoff, Self-similar asymptotics for linear and nonlinear diffusion equations, Stud. Appl. Math. 100 (1998) 153-193. 